\title{
De l'écrit vers la parole. Enquête sur les correspondances peu lettrées de la Grande Guerre
}

\author{
Steuckardt, Agnès \\ Université Paul-Valéry Montpellier 3 \\ Praxiling (UMR 5267) \\ agnes.steuckardt@univ-montp3.fr
}

\section{Introduction}

Que savons-nous de la «parole » des Poilus ? Si le célèbre recueil Paroles de Poilus publié en 1998 par Jean-Pierre Guéno et Yves Laplume, nous a donné à lire des lettres écrites par les soldats de 14, c'est, dans leur titre, de parole au sens large de «discours » qu'il est question. Mais pour la parole au sens spécifique de «discours oral », nous n'avons pas de témoignage direct: les quelques scènes de guerre filmées que nous avons conservées sont muettes; les enregistrements d'époque gravent la parole des acteurs, des chanteurs, des hommes politiques, des professeurs; les «Archives de la parole» ont recueilli, par les soins de Ferdinand Brunot, de précieux exemplaires des parlers dialectaux des campagnes des Ardennes, du Berry et du Limousin ; mais elles n’ont pas enregistré le français tel qu'il se parlait aussi dans cette Troisième République encore essentiellement rurale du début du $20^{\mathrm{e}}$ siècle : il était en effet la langue de communication lorsqu'on s'adressait aux administrations, qu'on se rendait en ville, et il était bien sûr la langue apprise à l'école ${ }^{1}$. Les Poilus connaissent et parlent cette langue française, qu'ils l'aient acquise au berceau ou apprise à l'école ${ }^{2}$. À sa femme, l'un d'eux recommande, à propos de leur fils :

(1) Il faut parler Français à Roger c'est tout et tu sais ce que je t'ai toujour dit il faut lui apprendre le francais sa ne coûte rien et c'est plus chic (Alfred, 20/10/1914)

De cette parole-là, qui se pratiquait donc aussi dans l'intimité familiale, nous n'avons pas d'enregistrement ${ }^{3}$.

Quand les médiévistes ne craignent pas de partir à la recherche d'une parole perdue depuis plus d'un demi-millénaire avec pour seul secours le témoignage de l'écrit (Guillot, 2009 ; Marchello-Nizia, 2012 ; Rodríguez Somolinos, 2013 ; Wirth-Jaillard, 2013), ne serait-il pas pusillanime de s'abstenir d'en faire autant, alors qu'un siècle seulement nous sépare des locuteurs? Pour le début du $20^{\mathrm{e}}$ siècle, des enregistrements d'époque existent, même s'ils ne concernent pas les Poilus eux-mêmes; la description scientifique de la parole est alors en plein essor, avec notamment les travaux de l'abbé Rousselot, de Jules Gilliéron, de Maurice Grammont ou de Ferdinand Brunot. Dans quelle mesure pouvons-nous utiliser, en complément de ces ressources indirectes, méta- ou épilinguistiques, le témoignage de l'écrit, à l'exemple des médiévistes?

Pour retrouver quelque chose de la parole des Poilus, on propose d'utiliser dans la présente étude un corpus de correspondances familiales peu lettrées de la période 1914-1916 ${ }^{4}$. On cherchera dans un premier temps à argumenter le recours à une démarche inductive menant de l'écrit vers, non pas seulement l'oralité, c'est-à-dire les caractéristiques lexicales, syntaxiques et stylistiques de leur discours oral, mais plus largement vers la parole, le terme englobant pour nous usage parlé et dimension phonique. On montrera comment l'écrit peu lettré, plus spécifiquement, peut permettre cette démarche inductive.

\section{Un « parlé graphique »}

Au vieil antagonisme entre «l'écrit» et «l'oral» fait progressivement place un questionnement sur cette dichotomie, déjà esquissé par Françoise Gadet dans « Une distinction bien fragile : oral/écrit » (1996), ou par Claire Blanche-Benveniste, qui intitulait «L'écrit présent dans l'oral» une partie du chapitre «Le 
parlé et l'écrit », d'Approches de la langue parlée en français ([1997] 2000 : 10-12). Peter Koch et Wulf Österreicher posent clairement la nécessité de distinguer d'une part «l'aspect médial » d'un énoncé, d'autre part son « aspect conceptionnel » (2001: 584). Si « l'aspect médial », qui concerne l'utilisation du chenal, soit écrit, soit oral, oblige à poser une dichotomie entre code écrit et code oral, en revanche «l'aspect conceptionnel », qui concerne le type de communication (de la " conversation spontanée entre amis » au «texte de loi », pour reprendre leur illustration des pôles extrêmes), est de nature scalaire. En croisant les options « conceptionnelles» (autrement dit les genres discursifs, du plus «parlé » au plus " écrit ») et «médiales » (c'est-à-dire les codes, oral et écrit), Koch et Österreicher identifient, « quatre combinaisons possibles: "parlé phonique", "parlé graphique", "écrit phonique", "écrit graphique" " (2001: 585). Dans la représentation spatiale donnée par les auteurs, «parlé phonique» et "écrit phonique " se placent respectivement en secteur A et B aux extrêmes opposés d'une ligne continue orientée selon une distance communicative croissante, tandis que " écrit phonique » et « écrit graphique » sont placés en secteur $\mathrm{C}$ et $\mathrm{D}$, au-dessus de cette ligne : une proximité entre "parlé graphique » (secteur C) et «parlé phonique » (secteur A), tous deux du côté de la faible distance communicative, est ainsi mise en évidence. Du point de vue « conceptionnel », la correspondance familiale des Poilus, qui se situe plus près d'une conception parlée que d'une conception écrite de la communication, relève, selon cette catégorisation sur laquelle on reviendra dans un premier temps, du " parlé graphique ».

\title{
2.1 Des chenaux et des genres
}

L'essentialisation de ce qui serait la «langue parlée » d'un côté, «la langue écrite » de l'autre semble relativement récente en français, dans la mesure où ces lexies ne commencent à être employées qu'au début du $18^{\mathrm{e}}$ siècle. $\mathrm{Si}$, chez les remarqueurs classiques, une opposition et une hiérarchie existaient entre l'usage de la conversation et l'usage écrit, c'est avec la description du chinois, et de ses caractères non phonogrammiques, que semble s'installer l'idée d'une différence non pas de registre, mais de nature entre 《langue parlée » et «langue écrite ${ }^{5}$; cette dichotomie n'a pas été sans provoquer, par le passé, des perplexités (Koch et Österreicher, $2001: 584$ ) ; à la fin du $20^{\mathrm{e}}$ siècle, des linguistes se sont employés plus systématiquement à la déconstruire, à l'exemple de Biber (1988), qui définit des genres transcendant l'opposition oral vs écrit, ou de Koch et Österreicher (2001), qui, plutôt qu'une solution de continuité entre écrit et oral, posent un continuum des réalisations discursives, distinguées en mesurant leur degré de distance communicative. La différence de nature entre «langue orale» et «langue écrite» peut désormais être contestée :

\begin{abstract}
Ce qui différencie l'oral de l'écrit (ou plutôt, les genres oraux des genres écrits), ce n'est pas tant la nature des constructions attestées, que les rendements qui leur sont assignés. Les conditions de production de chaque genre discursif étant différentes, elles induisent des modes d'optimisation et de coopération différents, d'où les préférences, parfois statistiquement sensibles, en faveur de certaines structures. Mais ces différences d'opportunité mises à part, leur grammaire a tout l'air d'être la même. Cf. [Blanche-Benveniste 1997: 65], [Gadet 1997: 52], [Béguelin 1998], [Berrendonner 2004]. (Berrendonner et Béguelin, 2012 : 19)
\end{abstract}

Le passage est placé en note, s'adosse à de solides autorités, et modalise l'affirmation d'identité leur grammaire a tout l'air d'être la même par la prudente périphrase a tout l'air. Mais la légitimité des expressions mêmes «l'oral» et «l'écrit» est mise en question, puisque les auteurs les corrigent, par le connecteur ou plutôt, en, respectivement, les genres oraux, les genres écrits. Une même grammaire donc, avec des "modes d'optimisation et de coopération différents", d'où des préférences pour certaines structures quand c'est le chenal oral qui est emprunté. L'analyse peut être étendue sans difficulté au lexique : un même lexique, avec des différences statistiques imputables aux genres discursifs, et non au chenal. Dès lors qu'il s'agit de la même langue, il semble loisible de chercher dans les productions écrites les caractères de genres relevant du «parlé » (et réciproquement, mais cette piste ne sera pas abordée ici). Les médiévistes se sont engagés dans cette voie en recherchant dans l'écrit médiéval les «traces d'oralité » (Guillot, 2009), les «marques d'oralité » (Rodríguez Somolinos, 2013), la « représentation de l'oral » (Wirth-Jaillard, 2013). Si l'on transpose leur démarche à la période plus récente du début du $20^{\mathrm{e}}$ 
siècle, on regardera d'abord, en s'inspirant de leur expérience, l'écrit des correspondances de la Grande Guerre comme un témoignage du «parlé », appréhendé en tant que genre.

\subsection{Correspondances de guerre peu lettrées et proximité communicative}

Sans doute existe-t-il « une relation privilégiée entre les aspects médiaux et les aspects communicatifs des productions langagières, l'écrit étant par nature plutôt associé au pôle de la distance communicative, l'oral étant au contraire naturellement plus proche de la proximité communicative » (Guillot, 2009), mais cette description prototypique peut être modulée et appréciée, selon les dix critères mis en évidence par Koch et Österreicher, à savoir : 1) communication privée vs communication publique ;2) interlocuteur intime $v s$ interlocuteur inconnu ; 3) émotionnalité forte vs émotionnalité faible ; 4) ancrage actionnel et situationnel $v s$ détachement actionnel et situationnel; 5) ancrage référentiel dans la situation $v s$ détachement référentiel de la situation ; 6) coprésence spatio-temporelle vs séparation spatio-temporelle ; 7) coopération communicative intense vs coopération communicative minime; 8) dialogue $v s$ monologue ; 9) communication spontanée vs communication préparée ; 10) liberté thématique $v s$ fixation thématique. Si l'on regarde les critères 1, 2, 3, 4, 5 et 10, notre correspondance se situe en effet du côté de la faible distance communicative.

Il s'agit d'une communication privée, s'adressant à un locuteur intime, correspondance entre le Poilu et sa femme, ses parents, son enfant, ou des proches (un frère, une belle-sœur) (critères 1 et 2). Les lettres et cartes sont empreintes d'une vive émotionnalité (critère 3) étant donné la situation de danger permanent où se trouvent les soldats. Elles sont traversées par l'angoisse de l'attente :

(2) Nous n'avons pas eu de tes nouvelles depuis lundi sa commmence a faire un peu long au mon Dieu comme elle est l'ongue cette semaine (Marie, 20/09/1914)

Il y passe parfois de la colère :

(3) C'est affreut; de voir un carnage comme nous avon c'est méme indigne détre ou nous somme (Laurent, 07/06/1915)

Le discours est fortement ancré dans le vécu, le temps, le lieu des scripteurs (4 et 5):

(4) jai fait un petit trou pour me mettre a labri des obus je fais la lettre alonger par terre, la terre et un petit carnet me serve de bureau (Laurent, 09/09/1915)

Le texte progresse avec une grande liberté thématique (critère 10), usant fréquemment du coq-à-l'âne. On passe par exemple ici du projet d'écriture au menu du dîner, puis au thème récurrent des photographies promises :

(5) Je veux aussi écrire a Narcisse Houillet ainssi qu'au cousin Joseph Pelar mais aujourd'hui je n'aurai pas le temps mais a la premiere occasion. Ce soir je vais manger une salade avec mon cammarade après la soupe tu me demande s'il est sur la photographie il y est comme toute l'Escouade (Alfred, 11/12/1914)

Entre le premier et le deuxième thème, la rupture est totale : le scripteur saute d'une idée à une autre ; entre le deuxième et le troisième, le glissement est un peu moins brutal : le pronom personnel $i l$ reprend en anaphore mon cammarade; l'abandon brutal du thème du dîner produit cependant une fracture textuelle.

Cette correspondance, comme toute correspondance, se distingue de la conversation privée par le critère 6 : les correspondants ne sont pas, de fait, en coprésence ; on remarquera cependant toute une stratégie discursive visant, chez le correspondant, à s'abstraire de sa situation d'énonciation réelle pour se projeter dans un espace/temps fictif où les correspondants seraient en coprésence, comme Pierre et sa femme Marie :

(6) Bien chèr Epoux

C'est après une journée de fatigue chéri que je viens passér un petit moment au près de toi (Marie, 14/09/1914) 


\begin{abstract}
Ma tres chère Epouse
C'est apres avoir manger la soupe que je viens passer un petit momment auprès de vous tous. Sur tout auprès de toi chérie ce petit instant m'et si précieux (Pierre, 8/10/1914)
\end{abstract}

Le verbe de mouvement venir permet au scripteur de s'extraire de son espace pour se déplacer «auprès » de son destinataire, et partager avec lui un « petit moment », « ce petit instant... si précieux ».

Qu'en est-il en effet de la coopération communicative (critère 7) ? L'intensification de cette coopération par le non verbal et le paraverbal des interactions en coprésence ne semble guère possible; le scripteur peut cependant mettre en œuvre une forme de coopération par l'usage de marqueurs discursifs tels que $t u$ sais :

(7) Au sujet de Louis tu sais nous somme bien dacord mais il faut le dire bien vite (Laurent, 26/02/1915)

Je me suis fait porter malade $\mathrm{j}$ 'avait mal au ventre tu sai comme sa me prenait quelque fois (Alfred, 18/05/1915)

Quelle est cette affaire «au sujet de Louis» dont parle Laurent ? Quel est ce «mal de ventre» dont souffre Alfred ? Pour construire le sens référentiel de au sujet de Louis et de mal au ventre, les scripteurs font appel au savoir qu'ils partagent avec leur correspondante : cette coopération est si nécessaire qu'un tiers lecteur ne peut accéder au sens exact de ces expressions. Malgré la séparation physique, une forme de coopération communicative existe, par le recours mémoriel à l'expérience commune, dans cette correspondance, comme ce peut être le cas aussi plus généralement dans les correspondances privées. L'emploi de la deuxième personne dans le marqueur discursif $t u$ sais nous invite de même à nuancer le caractère monologal de ces textes (critère 8). Bien sûr, le scripteur tient seul le crayon-plume, mais par le truchement du discours rapporté, il met en scène une situation dialogale :

(8) Tu me dis que j'ai du être contant oui je lai été jai été plus que contant car j'aurais pleuré de joie (Pierre, 15/10/1914)

Vous me dite de vous dire çi j'ai reçu le colis ébien oui je les mis dans mon sac (Laurent, 16/11/1914)

$\mathrm{Tu}$ me dememde la droit ou nous somme ébien je vai te le dirre nous somme dans le pas-de-Calais (Laurent, 04/12/1914)

Le texte est monologal sans doute, mais il fonctionne en « diaphonie effective » (Roulet, $1993: 86$ ). Non seulement il reprend le discours effectivement tenu par le correspondant, cité en discours indirect (que j'ai dû être contant / de vous dire çi j'ai reçu le colis / la droit ou nous somme), mais il y répond en utilisant des formes caractéristiques de l'interaction orale, comme le mot-phrase oui, l'interjection eh bien, et en mettant en œuvre ses modalités d'enchaînement ordinaires : la reprise lexicale (de contant dans la première citation, de nous somme dans la troisième) ou l'anaphore pronominale (le pronom personnel élidé $l$ - agglutiné dans lai -, anaphorique de contant dans la première citation; le même pronom $l$ agglutiné cette fois dans «les»- anaphorique de le colis dans la deuxième; le pronom le, anaphore conceptuelle de la question où êtes-vous?).

Qu'en est-il du neuvième critère : quel degré de préparation les scripteurs accordent-ils à leur texte ? Quand ils ne sont pas au feu, les soldats ont le temps de songer à ce qu'ils vont écrire ; on le perçoit par exemple dans cette mention d'une pensée de la veille :

(9) J'ai pensé a une chose hier qui pourrait m'être utile dans quelques temps (Alfred, 23/05/1916)

Cependant, sur les six occurrences du participe pensé, trois se trouvent dans des énoncés négatifs, du type : je voulais te dire ces derniers jours de m envoyer de largent je n'y ai pas pensé il a fallu ta lettre de ce soir pour m'y faire repenser (Pierre, 12/07/1915). La lettre est souvent quotidienne, parfois bi-quotidienne, ce qui ne laisse pas vraiment le temps d'une préparation approfondie. Pour autant, le scripteur n'évolue pas dans la même temporalité que le locuteur, et son texte ne présente généralement pas les disfluences, inachèvements, reprises caractéristiques d'un discours passant par le chenal oral. Exceptionnellement, on relève un inachèvement qui évoque la production orale : 
(10) Antoinette ma vez mi la dresse de son mari je croi que ces A je sais son non mes je ne me rapelle pas du numéro du secteur (Laurent, 16/02/1915)

L'écriture suit apparemment ici le travail mémoriel en train de s'accomplir pour retrouver l'adresse oubliée: l'énoncé je croi que ces reste en suspens. À la place de la séquence de présentatif, une interjection $A$, marquant l'agacement du soldat devant une défaillance de sa mémoire (je sais son non mes je ne me rappelle pas du numéro de secteur). Ce passage ressemble à l'imitation littéraire de l'oral : le scripteur a-t-il organisé cette mise en scène de son discours ? La plume a-t-elle suivi le mouvement de la parole intérieure? Le cas reste trop isolé pour que l'on puisse extrapoler.

Le phénomène de détachement, pratiqué à l'écrit, mais dont la fréquence plus haute est habituellement notée pour le discours oral ${ }^{6}$, est plus largement représenté :

(11) C'es malheureuse l'etre elles narrivait jamais (Marie, 03/10/1914)

A Elise j'y ecrirai de nouveau (Pierre, 10/01/1915).

Le tabac ne le fait pas trop voir (Louise, 09/03/1915)

Les autres il les à vendus 5 sous pieces (Alfred, 23/11/1914)

Comme dans la parole vive, le scripteur pose le thème de son énoncé et l'intègre, dans un second temps, à une structure syntaxique élaborée en le reprenant par un pronom personnel anaphorique. Ces reprises sont-elles préméditées ${ }^{7}$ ? Suivent-elles le mouvement de la pensée ? Si le genre de la correspondance familiale suffit à situer les textes du côté du "parlé graphique », la faible connaissance des usages écrits qu'ont leurs scripteurs permet de se demander si la proximité avec les usages oraux qu'ils présentent résulte d'une imitation construite ou d'une transposition spontanée.

\section{Une porosité spécifique de l'écrit peu lettré ?}

Les caractères stylistiques prototypiques du "parlé », dont la dislocation, évoquée ci-dessus, est un exemple, mais dont le corpus présente bien d'autres illustrations ${ }^{8}$, sont-ils imputables à l'inscription, consciente, dans un genre parlé ? Ou doivent-elles être mises au compte d'une proximité spécifique de l'écrit peu lettré au code oral? Les deux hypothèses peuvent être soutenues, et l'on penchera en faveur de l'une ou de l'autre suivant le degré d'aisance que l'on perçoit chez le scripteur, et suivant sa performance du moment.

\subsection{Différents degrés de porosité}

Parmi les scripteurs, nous pouvons distinguer par exemple entre Joséphine, qui écrit peu, dont les lettres sont brèves et présentent des hésitations sur les correspondances phonogrammiques ${ }^{9}$, et Pierre qui utilise un vocabulaire parfois soutenu et des structurations syntaxiques élaborées. Lorsque Joséphine écrit :

(12) Il fait tous se que lonve quil face des grimase il a fait a mamé et a tou lemonde de la maison (Joséphine, 19/02/1915)

on peut interpréter des grimase il a fait comme une dislocation à gauche directement calquée de l'usage oral.

En revanche lorsque Pierre écrit :

(13) Ce bonheur nous l'attendons cherie et Dieu voudra bien nous le donner (Pierre, $08 / 10 / 1914)$

la dislocation à gauche ce bonheur peut passer pour un effet de style: elle participe d'une certaine emphase rhétorique que l'on remarque chez ce scripteur. On n'interprètera donc pas de la même manière la dislocation chez l'une et chez l'autre : porosité de l'écrit dans le premier cas, recherche d'expressivité dans le second. Les inductions que l'on peut tirer de l'écrit peu lettré, parce que ce ne sont que des inductions, requièrent des précautions méthodologiques: ces indices demandent à être recoupés, confirmés, étayés. Par le passé, l'exploitation de ce type de ressources a pu susciter des réserves qui engagent le linguiste contemporain qui se risquerait sur cette voie à la plus grande prudence. 


\title{
3.2 Les objections de Meillet
}

Faute de précautions, Henri Frei s'était attiré les critiques acerbes d'Antoine Meillet. Pour sa Grammaire des fautes, il avait en effet utilisé notamment les lettres peu lettrées adressées à la Croix-Rouge de Genève au sujet des prisonniers de guerre, et affirmait avec un bel optimisme : "ces lettres reflètent assez fidèlement l'état de la langue courante et populaire d'aujourd'hui » (Frei, 1929: 37). Dans le compte rendu qu'il fait de l'ouvrage, Meillet non seulement lui reproche le caractère disparate et peu référencé de ses données, mais surtout considère que l'écrit peu lettré n'est pas une source d'information fiable sur la langue :

\begin{abstract}
Monsieur Frei a notamment tiré parti des lettres écrites à la Croix-Rouge à Genève au sujet des prisonniers de guerre ; il est malaisé de trouver matériel moins probant ; déjà les lettres d'illettrés adressées à des illettrés ne fournissent au linguiste que des données médiocres ; mais quand les illettrés écrivent à un bureau, on est devant des gens affolés par une tâche dépassant leur force et qui ne savent pas ce qu'ils font. (1930:147)
\end{abstract}

Cette critique (reprise et étayée par Gadet, $1998: 62-63$ ) vaut-elle pour le corpus ici analysé ? Notons d'abord que les scripteurs que nous étudions s'adressent à leur famille, et non à des instances officielles, ce qui minimise la tentation de l'hypercorrection. Meillet cependant regarde également les «lettres d'illettrés adressées à des illettrés » comme des "données médiocres", même si c'est à un degré moindre. Selon lui, même si le scripteur peu lettré ne cherche pas particulièrement à écrire une langue qu'il ne maitrise pas, il «s'embrouille» dans sa pratique de l'écrit. Meillet y voit dans ses productions plutôt de la gaucherie qu'un fidèle reflet de la langue qu'il parle : "d'une manière générale, nombre de faits signalés par l'auteur proviennent simplement de ce que la langue observée est celle de gens qui s'essaient gauchement à employer des termes et des tours qu'ils connaissent mal : vénéneux et un mot latin, et l'm de venimeux ne s'explique pas immédiatement par venin; le sujet populaire qui s'embrouille dans vénéneux et venimeux ne fait pas du « français avancé ; il gâte, par ignorance, une distinction fine et utile » (Meillet, $1930: 148)$.

Sans doute les scripteurs peu lettrés sont-ils parfois gauches dans leur usage de la langue. Dans notre corpus, on remarque cette gaucherie lorsque les scripteurs ont affaire à des termes qui ne leur sont pas familiers, ainsi les termes récemment appris aluminium, ou zeppelin :

(14) jai voulu te faire une bague en aluminion boche (Ernest, 12/05/1915)

il me demande duluminion se $\mathrm{n}$ est pas facile den avoir (Ernest, 01/06/1915)

mais j'ai pas plus guerre d'aluminiume il faut que j'en fonde (Alfred, 24/02/1916)

Nous avons aussi abattu un Zeplin et plusieurs avions c'est Toujours autant de moin (Alfred, 24/02/1916)

Ils «s'embrouillent» dans l'emploi d'expressions prêtant à ces «distinction[s] fine[s]» dont parle Meillet, comme imprévu et à improviste :

(15) lautre jour nous avons mis des colets et des pièges juste nous somes partis a linprevu il nous a falu quitter le même jour que nous les avons mis au même (Ernest, 29/01/1916)

Mais ces gaucheries ne sont pas nécessairement l'indice d'un malaise spécifiquement provoqué par l'usage du code écrit : elles peuvent apparaitre aussi comme un témoignage de la parole des peu lettrés, avec ses approximations; elles ne sont d'ailleurs pas très abondantes dans notre corpus ${ }^{10}$. Loin de la représentation de "gens affolés » que propose Meillet, il se dégage la lecture de ces correspondances celle de scripteurs conscients du caractère vital du lien communicationnel que constitue la lettre, et assez indifférents aux préoccupations normatives, dérisoires dans un tel contexte ${ }^{11}$. Le corpus étudié, référencé avec précision, peu marqué par l'hypercorrection, guère plus "gauche » que ne peut l'être le parlé phonique, ne nous semble pas pouvoir prêter le flanc aux critiques portées par Meillet sur celui d'Henri Frei. L'exploitation que l'on en propose est aussi plus circonspecte : on ne prétendra pas que ce corpus, si particulier, « reflète assez fidèlement l'état de la langue courante et populaire » (Frei, cité supra), mais on estime pouvoir en extraire des indices non seulement sur l'usage parlé conçu en tant que genre, à la 
manière de ce qui se pratique dans les études des médiévistes, mais aussi sur ce qui relève du domaine propre de la parole vive : la prononciation.

\subsection{L'écrit peu lettré, témoignage de la prononciation}

Chez le scripteur peu lettré, la connaissance partielle de l'orthographe standard peut laisser supposer une notation syllabique et une pratique directe de correspondances phonogrammiques. Des exploitations de l'écrit peu lettré pour trouver trace de la prononciation ont déjà été proposées notamment par Sonia Branca-Rosoff et Nathalie Schneider, qui cherchaient dans les textes des peu lettrés révolutionnaires de Provence une « influence de la prononciation» méridionale (1994: 50-53) et par France Martineau, qui s'appuie sur le Journaille Commansé le 29 octobre 1765 de Charles-André Barthe pour tenter de reconstruire certaines prononciations québécoises du $18^{\mathrm{e}}$ siècle $(2010$ : 23-25). Comme le souligne France Martineau, les informations que l'on peut tirer des écrits peu lettrés demandent à être confirmées par la « régularité de la graphie », par son « attestation dans d'autres textes de la région », par des « traces dans le français actuel » (2010: 23), ainsi que par d'éventuelles descriptions épi- ou métalinguistiques. Pour peu que l'information donnée par l'écrit peu lettré puisse être recoupée, elle mérite attention. Notre corpus étant constitué, pour 75\%, de scripteurs héraultais (Ernest, Joséphine, Laurent, Louise, Pierre, Marie) et, pour 25\%, de scripteurs de l'Ain et de la Marne (Alfred, André, Auguste, Émile), nous donnerons deux types d'exemples : les premiers sont repérables sur l'ensemble du corpus, les seconds spécifiques des scripteurs languedociens.

\subsubsection{Des traits partagés}

La segmentation pratiquée par les scripteurs témoigne, à des degrés divers, d'un conflit entre perception syllabique, conscience sémiotique du mot, connaissance de graphies normées. Les classes grammaticales les plus sujettes à la notation syllabique sont, classiquement, les articles, les prépositions, les pronoms conjoints (Beguelin, 2003 : 7), comme dans lautre (pour l'autre, exemple 15), jai pour j'ai et les pour l'ai (exemple 8); il en va de même de la conjonction que, avec par exemple la graphie quil (exemple 11), ou le discordanciel $n e$, quand ils sont suivis d'une voyelle. Est en effet très généralement partagée la forme $n a$ (277 occurrences), qui soude le discordanciel élidé $n$ ' et la troisième personne verbe avoir, comme dans : il na pas voulu (Marie, 9/10/1914), ou le pronom en non nasalisé (cf. 3.3.2.), comme dans : je na sai rien (Laurent, 25/02/1915). Pour ces mots-outils, la perception syllabique peut donc l'emporter, malgré l'apprentissage scolaire.

Les mots pleins donnent parfois lieu aussi à des découpages syllabiques, comme on a pu le remarquer avec la segmentation ma vez (pour « m'avez », exemple 10), ou avec la soudure lonve pour l'on veut, en (12). Les prénoms, qui n'ont probablement pas fait l'objet d'un apprentissage scolaire, laissent apercevoir les conflits qui se jouent dans les réalisations graphiques. Le prénom Antoine donne lieu par exemple à la réalisation graphique suivante : «je de dirai quen entoines Entoine Rey et enpermision dun mois » (Joséphine, 13/01/1915), où l'on voit d'abord notée la concaténation quen, puis une forme autonome entoines; Joséphine semble ensuite se rappeler la majuscule due au nom propre et rature pour écrire finalement Entoine. Dans la succession de ces actes d'écriture, on suit les tentatives pour se dégager de l'écoute syllabique et se rapprocher de la graphie normée. La direction est plus incertaine dans la recherche menée par un autre des scripteurs, aux prises avec la graphie du prénom Eléonore, qui apparaît neuf fois dans sa correspondance :

(16) ja n'est eu par et l'Eonore (Laurent, 16/02/1915, à sa femme et à ses parents) jan es eu de l'Eore Elèonore (Laurent, 16/02/1915, à ses beaux-parents) j'ai aussi reçu une lettres des l'Eonore (Laurent, 01/03/1915) on i donnera tous les renseignement vou lu comme j'ai di a l'Eonore la cousine (Laurent, 22/03/1915)

j'écri a Eléonore en même temp qua vous autres (Laurent, 30/03/1915)

Les quatre occurrences ultérieures conservent la graphie Eléonore. La comparaison des deux premières graphies, l'une réservée au foyer, l'autre aux beaux-parents, semble montrer que la forme normée est 
connue et disponible quand le scripteur surveille la conformité à la norme. Mais, lorsqu'il écrit pour les proches, l'instabilité des graphies de Laurent semble indiquer une fragilité dans la prononciation de la voyelle initiale, clairement absente de la séquence j'ai di a l'Eonore du 22/03/1915. Cette probable fragilité le conduit à chercher des solutions mobilisant des mots graphiques vraisemblables - et suivi de $l$ ' (dans et l'Eonore, 16/02/1915), des suivi de l' (dans des l'Eonore, 16/02/1915) - tout en préservant la majuscule du prénom. La variation dans la segmentation graphique témoigne donc, chez les scripteurs peu lettrés, d'une perception de discontinuités, de liaisons, d'ellipses qui sont celles de la chaîne parlée. Plus spécifiquement, on peut chercher dans les correspondances phonogrammiques qu'ils proposent des indices de leurs manières de prononcer.

Le $e$ est absent chez les scripteurs du nord et du sud dans le mot caleçon (15 occurrences de calçon(s)/calcons, pas une seule de caleçon(s)) ; on relève également tu fras (Ernest, 31/01/1915). Selon Grammont, dont le Traité de prononciation est contemporain, « quand il n'est séparé de la voyelle qui le précède que par une seule consonne, [le e caduc] tombe toujours » ([1914] 1951:115; voir aussi Bauche, 1920 : 36-37). En revanche, sur d'autres mots, comme petit, ce type d'amuïssement n'est pas visible dans le corpus, probablement parce que la graphie de ce mot est trop bien connue des scripteurs.

Le pronom $i l$ est fréquemment réduit à $i$ (Grammont, [1914] $1951: 95)$ :

(17) je veut pourtant demander un calçon quand le mien sera sal comme i n'est pas neuf je le balancerai (Alfred, 21/10/1914)

Il vaut mieux qui soit degourdie (Louise, 02/04/1915)

Si l'influence de la prononciation ne fait pas de doute pour ces graphies, on hésitera sur l'interprétation des doubles $r$ : transcrivent-ils ce $r$ roulé encore décrit dans la prononciation non parisienne du début du siècle (Grammont, [1914] 1951: 66), ou bien une incertitude orthographique ? Le verbe dire, le nom caresse s'écrivent ordinairement chez les scripteurs du sud avec deux $r$ : peut-être s'agit-il d'une habitude orthographique. Alfred (scripteur de l'Ain) écrit berret, parraissent (1 occurrence), parrait (2 occurrences mais 8 fois parait), derranger (mais il peut y avoir analogie avec arranger), guerrison, guerrit, guerrite (mais le modèle de guerre peut interférer); on remarquera qu'il écrit trois fois Harraucourt (pour deux occurrences de la graphie normée Haraucourt), alors que les toponymes, que les soldats pouvaient lire sur les panneaux, sont généralement orthographiés fidèlement. Il semble que l'on puisse pencher en faveur de l'hypothèse de la transcription d'une prononciation, lorsqu'il ne s'agit pas de lexème stabilisé :

(18) dans des condition com ça tout le monde arrefuser de marchez, et plus sa yra, personne ne voudra monter (Laurent, 02/11/1915) mai au sujet des lettres en corre je nai rien reçu (Laurent, 05/12/1915)

Ici Laurent ne segmente pas la forme composée a refusé : il ne semble pas identifier chaque mot, mais noter ce que sa parole intérieure lui dicte; de même la graphie en corre paraît indiquer que le mot encore n'est pas conçu comme une unité lexicale, mais que le scripteur note les phonèmes qu'il entend.

\subsubsection{Prononciations dialectales}

Parmi de nombreux petits faits attestés dans le corpus, on présentera ici deux exemples de prononciation, l'une largement attestée, l'autre très localisée.

Chez l'ensemble des locuteurs de l'Hérault, on remarque une utilisation fréquente de graphie $a$, là où l'on attendrait an ou en :

(19) Il la n'est de meme pour moi (Laurent, 28/09/1914) (59 occurrences de la formule chez ce locuteur)

ja nai pas eu (Laurent, 28/09/1914); ja nai envoyez (22/12/1915) (45

occurrences de ja suivi de nai/n'est/navez)

on sa nai tirer une foi (Laurent, 01/10/1914)

ses le payre Reynaux du Bos quil te la vois (Joséphine, 13/01/1915)

il a net de meme pour nous (Joséphine, 19/02/1915;23/02/1915) 
De même, Laurent écrit la droit pour signifier «l'endroit » (8), et on relève une graphie $e$ pour le mot standard instant:

(20) a lestent même je reçois ta chere lettre (Ernest, 29/01/1816)

Ces graphies récurrentes et attestées chez plusieurs scripteurs indiquent une nasalisation incomplète, repérée et décrite en termes normatifs par Grammont :

Très défectueuses aussi sont les dénasalisations ${ }^{12}$ méridionales, telles que anivré « enivrer », an $\ddot{w} i$ « ennui » au lieu de ãn $\ddot{w}$. ([1914] $1951: 56)$

Cette nasalisation incomplète méridionale est relevée aussi dans les écrits peu lettrés de la période révolutionnaire étudiés par Sonia Branca-Rosoff et Nathalie Schneider (1994 : 55).

Plus localement, on remarque chez les trois scripteurs qui habitent le village du Soulié (ouest de l'Hérault) une graphie $s$ pour l'initiale du verbe changer et de ses dérivés :

(21) je compte passer le conseil vers la fin de ce mois si je ne sange pas d'hopital peut-être avant. (Pierre, 08/10/1914)

Pas de sangement chez nous (Ernest, 18/05/1915)

$\mathrm{Si}$ ca continuet ca irait bien mai peut etre il sangera de nouveau car il sange souvent (Marie, 01/02/1915)

le 1er jour nous navons fait que $12 \mathrm{~K}$ le deuxieme jour sa a sangé nous avons fait dans les $32 \mathrm{~K}$ (Ernest, 29/01/1916)

L'enquête auprès de locuteurs âgés de ce village a permis de confirmer cette prononciation locale ; cette dissimilation des chuintantes se retrouve dans d'autres parlers dialectaux ${ }^{13}$.

\section{Conclusion}

Tirer de l'écrit peu lettré des informations sur la manière de parler d'une époque, d'une région, d'un village, est une entreprise qui doit s'entourer de précautions; ainsi par exemple, pour les graphies, les fonctionnements analogiques et iconiques propres à la scripturalité doivent-ils être pris en considération. Si les graphies des peu lettrés sont moins corsetées que celle de l'écrit standard, il ne s'agit évidemment pas d'une notation fidèle de leur prononciation et de leur verbe.

$\mathrm{Au}$ niveau discursif, notre corpus de correspondances n'est pas exempt des routines épistolaires mémorisées et restituées ; pour autant, les scripteurs ne semblent pas tétanisés par le medium écrit et, une fois passée la formule stéréotypée d'ouverture, ils improvisent une prose personnelle, choisissant par exemple des marqueurs discursifs qui leur permettent à la fois de rythmer leur discours et d'y installer une diaphonie différée.

$\mathrm{Au}$ niveau phonogrammique, on relève des cas d'hypercorrection et l'image visuelle des mots appris intervient dans les productions écrites, mais, en l'absence de référent iconique, le scripteur réinvente un codage qui serre au plus près sa parole, nous livrant des informations précieuses sur son appropriation du français parlé, comme cette nasalisation incomplète décrite plus ordinairement chez les Provençaux que chez les Languedociens.

Koch et Österreicher, après avoir rappelé que « le latin vulgaire en tant que latin « parlé » n'est accessible qu'à travers des sources, telles que « certains types d'inscriptions » ou « des lettres de soldats », et qu' " il serait naïf de penser que ces témoignages reflètent fidèlement l'immédiat phonique », estiment néanmoins que «la linguistique variationnelle diachronique aurait grand tort de se résigner: tout en restant consciente des limites indéniables que comporte l'utilisation des textes du secteur C [ " parlé graphique »], elle a intérêt à élaborer les instruments nécessaires pour une analyse qui nous fournisse le maximum d'informations, certes en partie indirectes, sur la réalité historique du secteur A [ «parlé phonique »] » (2001: 615). Nous ferons nôtre ce point de vue, en soulignant que la linguistique variationnelle diachronique concerne non seulement le latin vulgaire, mais aussi le français qui se parlait il y a cent ans. 


\section{Références bibliographiques}

Baconnier, G., Minet, A., Soler, L. (1985). La plume au fusil : les Poilus du Midi à travers leur correspondance, Toulouse : Éditions Privat.

Bauche, H. (1920). Le langage populaire. Grammaire, syntaxe et dictionnaire. Paris : Payot.

Béguelin M.-J. (2002). Unidades de lengua y unidades de escritura. Evolucion y modalidades de la segmentación gráfica. Emilia Feirrero (éd.), Relaciones de (in)dependencia entre oralidad y escritura. Barcelone, Gedisa, Colleccion LeA, 51-71.

Berrendonner, A. et Béguelin, M.-J. (2012). Pour en finir avec la phrase. Groupe de Fribourg, Grammaire de la période. Bern : Peter Lang, 3-19.

Biber, D. (1988). Variation Across speech and writing. Cambridge : Cambridge University Press.

Blanche-Benveniste, C. (1997). Approches de la langue parlée en français. Gap/Paris : Ophrys.

Branca-Rosoff, S., Schneider, N. (1994). L'écriture des citoyens. Une analyse de l'écriture des peu-lettrés pendant la période révolutionnaire. Paris : Klincksieck.

Gadet, F. (1996). Une distinction bien fragile : oral/écrit. Tranel, $\mathrm{n}^{\circ} 25,13-27$.

Gadet, F. (1998). Le français avancé à l'épreuve de ses données. Mireille Bilger, Karel van den Eynde, Françoise Gadet (éds), Analyses linguistiques et approches de l'oral. Recueil d'études offert en hommage à Claire BlancheBenveniste. Leuven-Paris : Peeters, 59-68.

Gardy, P., Lafont, R. (1981). La diglossie comme conflit : l'exemple occitan. Langages, $\mathrm{n}^{\circ} 61$. Bilinguisme et diglossie, 75-91.

Grammont, M. ([1914] 1951). Traité de prononciation française. Paris : Delagrave.

Guéno, J.-P., Laplume, Y. (1998) Paroles de Poilus. Paris : Librio/Radio France.

Fourmont, É. (1735). Réflexions critiques sur les histoires des anciens peuples, t. 2. Paris : Musier père.

Fréret, N. ([1718] 1723). Réflexions sur les principes généraux de l'art d'écrire et en particulier sur les fondements de l'écriture chinoise. Mémoires de l'Académie des inscriptions et belles-lettres, IV. Paris : Imprimerie royale, 411-436.

Guillot, C. (2009). Écrit médiéval et traces d'oralité : l'exemple de l'adverbe or(e). E. Havu et al. (éds), La langue en contexte. Actes du colloque Représentation du sens linguistique IV (Helsinki, 28-30 mai 2008). Helsinki : Société Néophilologique, 267-281.

Koch, P. \& Österreicher, W. (2001). Gesprochene Sprache und geschriebene Sprache. Langage parlé et langage écrit. G. Holtus, M. Metzeltin et C. Schmitt (éd.), Lexikon der romanistischen Linguistik, 1-2, 584-627.

Heiden, S. (2010). The TXM Platform : Building Open-Source Textual Analysis Software Compatible with the TEI Encoding Scheme. In K. I. Ryo Otoguro (éd.), 24th Pacific Asia Conference on Language, Information and Computation - $\underline{\text { PACLIC24 }}$, 389-398.

Luxardo, G. (à paraître). Fréquence des colis et marmites : comment mesurer la languitude ? A. Steuckardt (dir.), Pour me lever de languir. L'écriture des Poilus. Toulouse : Éditions Privat.

Marchello-Nizia, C. (2012). L'“oral représenté” en français médiéval, un accès construit à une face cachée des langues mortes. C. Guillot, B. Combettes, A. Lavrentiev, E. Oppermann-Marsaux \& S. Prévost (éd.), Le changement en français. Études de linguistique diachronique. Bern : Peter Lang, 247-264.

Martineau, F. et Bénéteau, M. (2010). Incursion dans le Détroit. Journaille Commansé le 29 octobre 1765 de Charles-André Barthe. Laval : Presses de l’Université de Laval.

Meillet, A. (1930). Compte rendu de La grammaire des fautes de Henri Frei. Bulletin de la société de linguistique de Paris, 145-149.

Paris, G. (1898). Compte rendu de La dissimilation consonantique dans les langues indo-européennes et dans les langues romanes, de Maurice Grammont. Journal des savants, numéro de février, 81-99.

Prein, A. (1921). Syntaktisches aus französichen Soldatenbriefen. Giessen : Selbstverlag des Romanischen Seminars. 
Riegel, M., Pellat J.-C., Rioul, R. (1994). Grammaire méthodique du français. Paris : Presses Universitaires de France.

Rodríguez-Somolinos, A. (dir.). (2013). Diachroniques, n 3, Marques d'oralité en français médiéval.

Ronjat, J. (1913). Essai de syntaxe des parlers provençaux modernes. Mâcon : Protat Frères.

Roulet, E. (1993). De la structure diaphonique du discours épistolaire : à propos d'une lettre d'Aurore Dupin à sa mère. Mélanges offerts à Jean Peytard, Besançon, Annales littéraires de l'Université de Besançon, n 502, 85-99.

Rousselot, J.-P. (1911). Dictionnaire de la prononciation française. Revue de Phonétique, I, 169-180.

Wirth-Jaillard, A. (2013). "De Estienne Husson pour ce qu'il dit a Jannon Morelot : 'un filz de bastarde ne me puet valoir !"” : représentation de l'oral dans les documents comptables médiévaux. D. Lagorgette \& P. Larrivée (éd.). Représentations $d u$ sens linguistique V. Chambéry : Éditions de l’Université de Savoie, 65-80.

\footnotetext{
${ }^{1}$ L'article 14 de l'arrêté du 7 juin 1880 stipulait : « Le français sera la seule langue en usage à l'école ».

${ }^{2}$ Sur la situation de diglossie, voir notamment pour l'occitan Philippe Gardy et Robert Lafont, 1981. Pour leur correspondance, les Poilus utilisent le français : «Nos Méridionaux - Languedociens, Catalans et Provençaux s'expriment en français et non pas dans leur langue maternelle. Et nous n'avons trouvé que trois cartes postales entièrement rédigées en occitan ", écrivent les auteurs de La Plume au fusil: les Poilus du Midi à travers leur correspondance (1985: 19), qui ont dépouillé 6583 lettres et cartes postales. Notre corpus confirme ce constat. Quelques mots en occitan y apparaissent, très sporadiquement, comme l'injection rä̈ ("n'importe », "ça ira »), utilisée par Marie. La pratique écrite de l'occitan relève du militantisme, dont témoigne la correspondance du jeune félibre Louis Bonfils, redécouverte par Guy Barral (http://bibliophilelanguedocien.blogspot.fr/2012/02/le-journal-deguerre-en-occitan-dun.html, consulté le 9/03/2014).
}

${ }^{3}$ Il existe bien sûr des enregistrements ultérieurs des Poilus, réalisés surtout à partir des années 70 ; ils présentent cependant plusieurs biais, le principal étant le changement de la langue elle-même.

${ }^{4}$ Ce corpus comporte 650 lettres et cartes, écrites par 10 scripteurs et compte 157800 mots ; il est actuellement accueilli avec l'aimable collaboration de Serge Heiden et de son équipe (ICAR, UMR 5191), sur le portail TXM (Heiden, 2010). Depuis 2013, il est consultable en accès réservé à l'adresse http://portal.textometrie.org/demo/?locale=fr. Il sera accessible et pérennisé en 2014 sur l'Equipex ORTOLANG, à l'adresse : www.ortolang.fr.

${ }^{5}$ Les expressions de langue parlée et langue écrite, employées par Nicolas Fréret (Réflexions sur les principes généraux de l'art d'écrire et en particulier sur les fondements de l'écriture chinoise, 1718) et par Etienne Fourmont (Sur la littérature chinoise, 1722) pour décrire les usages linguistiques chinois, étonne encore en 1730: «Cette langue [le chinois] si singuliere en son espece, $\&$ ne ressemblant à aucune de celles que nous connoissons, doit être considerée sous deux points de vûe differens ; c'est à-dire, comme Langue parlée, s'il est permis de s'énoncer ainsi, ou comme Langue écrite" (Journal des sçavans, [Compte rendu de Sur la littérature chinoise, juin 1730, Amsterdam, Chez les Jansons à Waesberge : 209). La modalisation autonymique corrective $s$ 'il est permis de s'énoncer ainsi indique que la notion de Langue parlée paraît sinon maladroite, au moins inhabituelle à l'auteur anonyme du compte rendu.

${ }^{6}$ «Ces procédés [l'emphase par dislocation ou par extraction] sont très employés à l'oral » (Riegel, Pellat, Rioul, $1994: 426)$.

${ }^{7}$ Les lettres reçues du front donnaient lieu à lecture collective, comme en témoigne une des épistolières : « qu'ant le soir arrive (le soir Ernestou nous porte les letre et nous alons les prendre au soulié bas) que nous arivons du soulié bas que nous pouvons lire en famille une letre de toi chéri et de mon Frère tu peu croire mon bien Aimé que c'est un vrai bonheur pour ton épouse et pour tes chers parents (Marie, 03/02/1915).

${ }^{8}$ Ainsi l'absence du discordanciel ne, l'emploi du pronom on pour désigner une personne 4, ou l'emploi de ça. (277 occurrences). Selon l'évaluation que nous avons pu faire, dans les négations qui requièrent, en français standard, discordanciel et forclusif, les scripteurs de notre corpus omettent le ne dans $25 \%$ des cas. L'emploi du pronom on (sur l'approche quantitative des pronoms dans ce corpus, voir Luxardo, à paraître) est dominant chez les scripteurs de l'Ain avec 452 on, pour 362 nous ; chez les scripteurs de l'Hérault, la tendance est inverse, peut-être parce que, 
l'expression du pronom sujet étant moins nécessaire en occitan qu'en français (Ronjat, 1913: 14), ils sont plus attentifs à l'emploi du pronom appris. La forme ça présente quant à elle 277 occurrences, auxquelles il faut ajouter environ $15 \%$ des 284 graphies $s a$, employées majoritairement avec la valeur de déterminant possessif, mais aussi avec d'autres valeurs parmi lesquelles celle du pronom démonstratif.

${ }^{9}$ Ainsi, la préposition dans est graphié ten ou dent, la préposition de est graphiée te ou de.

${ }^{10}$ On peine en particulier à trouver des hypercorrections de nature syntaxique ; signalons : « on me les a faite [vos lettres] parvenir » (Laurent, 16/05/1916).

${ }^{11}$ On citera par exemple cette réflexion de Laurent : « çi je te fait des lettres courte c'est que depuis 13 mois je ne sais plus quoi mettre de sur mai toi étand dans un village elle serait un peu plus longue çeula ne gaterai pas, au puis écoute je suis obliger de les prendre comme elles sont » (Laurent, 27/08/1915).

${ }^{12}$ Dans ce type de cas, l'hypothèse d'une nasalisation incomplète est jugée plus vraisemblable par l'abbé Rousselot : «Est-ce chez moi une tendance à la dénasalisation ou nasalisation incomplète ? La seconde hypothèse est seule probable dans le voisinage du Midi » (Rousselot, 1911 : 177).

${ }^{13}$ Gaston Paris note la forme sanger dans une liste de dissimilation qu'il dresse à l'appui de la thèse de Grammont sur la dissimulation consonantique (1898: 95). 